\title{
Variant ataxia-telangiectasia in a child presenting with laryngeal dystonia
}

\author{
Pınar Arıcan ${ }^{1 \oplus}$, Nihal Olgaç Dündar ${ }^{2 \oplus}$, Özgür Kırbıyık ${ }^{3 \oplus}$, Dilek Çavusoğlu ${ }^{4 \oplus}$, \\ Sema Bozkaya Yılmaz ${ }^{1 \oplus}$, Pınar Gençpınar²ఠ \\ Departments of ${ }^{1}$ Pediatric Neurology and ${ }^{3}$ Genetic, Izmir Tepecik Training and Research Hospital, Izmir; ${ }^{2}$ Department of Pediatric \\ Neurology, Izmir Katip Celebi University, İzmir; ${ }^{4}$ Department of Pediatric Neurology, Afyon Kocatepe University Faculty of Medicine, \\ Afyon, Turkey.
}

\begin{abstract}
Background. Dystonia is a common hyperkinetic movement disorder in children; however, making an early and definitive diagnosis of dystonia can sometimes be challenging for clinicians.

Case. Herein, we report a case of a 16 years-old girl presenting with laryngeal dystonia due to compound heterozygosity of a known pathogenic and a novel variant in the ATM gene. Serum alpha-fetoprotein level was elevated. Serum IgG, IgA, IgM and IgE levels were within normal range. Treatment with L-DOPA had no benefit. Her symptoms were dramatically improved by localized botulinum toxin injections.

Conclusion. Mutations in the ATM gene show a wide phenotypic spectrum from severe classical early-onset ataxia-telangiectasia (A-T) to late-onset milder variant A-T. Our findings highlight the importance of recognizing laryngeal dystonia as one of the clinical signs of A-T.
\end{abstract}

Key words: ataxia-telangiectasia, ATM gene, dysarthria, dystonia.

Dystonia is one of the most common hyperkinetic pediatric movement disorders. It is defined as involuntary, twisting movements and abnormal posture, caused by sustained or intermittent muscle contractions. ${ }^{1}$ Clinical features of dystonia may be non-specific or atypical and can be diagnosed several years after the first symptoms manifest. ${ }^{2}$ Dystonia prevents the acquisition of normal motor skills during critical periods of development in children and has negative impacts on activities of daily living and quality of life. ${ }^{3}$

Primary dystonia consists of a genetic heterogeneous group of disorders with onset of symptoms during childhood or adolescence. Advances in genetic techniques in the past years have widened the etiological spectrum

Nihal Olgaç Dündar

nodundar@gmail.com

Received 18th July 2019, revised 13th September 2019, accepted 4th October 2019. of primary dystonia and other neurological conditions that can present as primary dystonia such as ataxia telangiectasia (A-T) and spinocerebellar ataxia type $3 .^{4-6}$

The typical phenotype is childhood onset of focal dystonia, mainly in the lower limbs and more likely that can spread to multiple body parts over time. ${ }^{7}$ Herein, we report a case of a 16 years-old girl presenting with laryngeal dystonia due to compound heterozygosity of a known pathogenic and a novel variant in the ATM gene.

\section{Case Report}

A 16-years-old female patient admitted with complaints of fatigability and dysarthria. She was the third child of non-consanguineous parents with no relevant medical familial history. She was born full term after a normal pregnancy. Her neuromotor development was normal until the age of 10 years when 
she developed fatigability and dysarthria. On physical examination, her weight, height, and head circumference were normal for her age. Neurologic examination was normal, except for dysarthria and facial weakness. There were no organomegaly and dysmorphic features.

Complete blood count, serum electrolytes, renal function, liver function, thyroid function tests and creatinine kinase level were normal. Cranial and cervical spine magnetic resonance imaging was normal. Myasthenia was suspected and further investigations were performed. Electromyography with repetitive nerve stimulation was normal. Acetylcholine receptor antibodies and MuSK antibodies were negative. Analysis of the CHRNE gene did not show a mutation.

For further genetic investigation of possible neurometabolic disease, TruSight Inherited Disease Sequencing Panel (Illumina Inc.) was used. She was found compound heterozygous for ATM variant (Fig. 1). The variant c.8147T>C (p.Val2716Ala) has been described ${ }^{7}$, whereas nonsense variant c.7424T>G (p.Leu2475Ter) has not been described so far, but is predicted in silico to be pathogenic. The unaffected parents were heterozygous for the variants. Sanger sequencing in parents showed the biallelic origin of the two variants, with the mother harboring the c.8147T $>\mathrm{C}$ (p.Val2716Ala) variant, and the father harboring the c.7424T>G (p.Leu2475Ter) variant (Fig. 1).

Serum alpha-fetoprotein level was $65.32 \mathrm{ng} / \mathrm{ml}$ (reference range: $0-5.8$ ). She had no clinical or laboratory signs of malignancy and abdominal ultrasound showed no abnormality. Serum IgG, IgA, $\operatorname{IgM}$ and $\operatorname{IgE}$ levels were within normal range. There was no history of recurrent sinopulmonary infections. At the age of eighteen, she developed oromandibular dystonia. Subsequently, treatment with L-DOPA was started at $4 \mathrm{mg} / \mathrm{kg} /$ day for a week and then the dose was increased to $8 \mathrm{mg} / \mathrm{kg} /$ day. Treatment with L-DOPA had no benefit. Oromandibular dystonia was dramatically improved by localized botulinum toxin injections into the genioglossus, mentalis, corrugator supercilii muscles with injection doses of 5 units and into the lateral pterygoid muscles with injection doses of 10 units. A written informed consent was obtained from the parents of the patient.

\section{Discussion}

We identified a variant form of A-T presented with laryngeal dystonia. A-T, due to mutations in the ATM gene, is a recessively inherited multisystem disorder. It is characterized by oculocutaneous telangiectasia, progressive cerebellar ataxia, choreoathetosis, recurrent sinopulmonary infections and an increased risk for cancer. ${ }^{8,9}$

Mutations in the ATM gene show a wide phenotypic spectrum from severe classical early-onset A-T to late-onset milder variant A-T. Clinical severity generally depends on the presence of ATM protein and kinase activity. ${ }^{10}$ In variant form of $\mathrm{A}-\mathrm{T}$, movement disorders appear to dominate the clinical presentation. Atypical clinical manifestations including lateonset focal or generalized dystonia, resting tremor and myoclonus have been described..$^{11-13}$

To date, the pathophysiology of dystonia in variant $\mathrm{A}$-T has not been well understood. Eilam et al. ${ }^{14}$ showed selective loss of dopaminergic nigrostriatal neurons in ATM-deficient mice and suggested that ATM deficiency could severely affect dopaminergic neurons in the central nervous system. Koepp et al. ${ }^{15}$ showed a decreased tracer uptake in the striatum bilaterally in a 6-year-old girl with A-T and severe progressive dystonia.

The ATM gene plays a central role in DNA damage responses and patients with A-T demonstrate increased sensitivity to ionizing radiation. Even though cases of variant $\mathrm{A}-\mathrm{T}$ have much milder neurological manifestations than in classic A-T, patients still have increased risk of malignancies. ${ }^{12}$ It is important to provide counseling and monitoring for neoplasms and to take measures in order to lessen accumulating DNA damage from radiologic exposure and chemotherapeutic agents. 

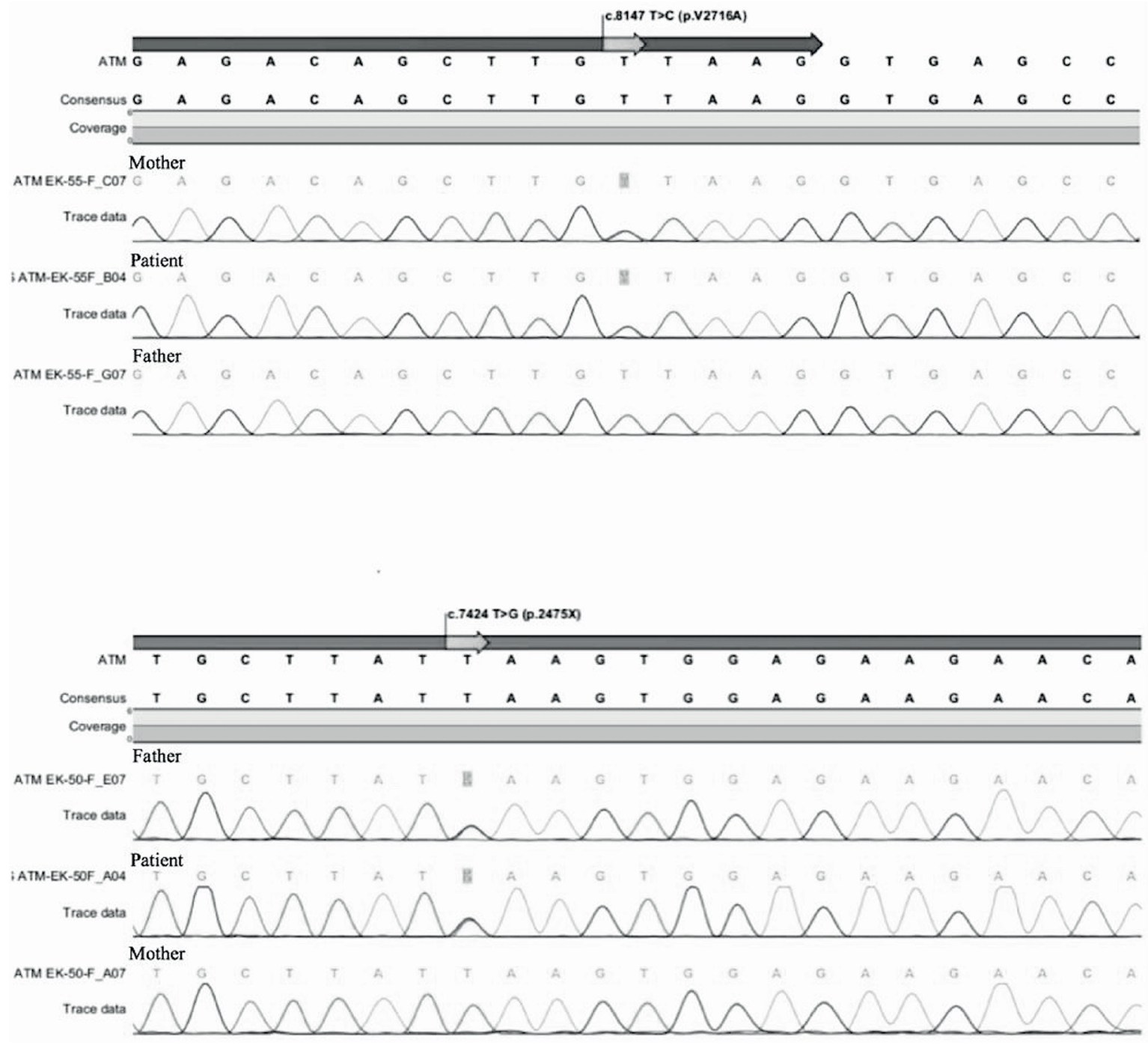

Fig. 1. Sanger sequencing in patient showed compound heterozygous variants, with the mother harboring the c.8147T $>$ C (p.Val2716Ala) variant, and the father harboring the c.7424T $>\mathrm{G}$ (p.Leu2475Ter) variant.

In patients with dystonia due to ATM mutations, treatment is challenging and efficacy is typically incomplete. Botulinum toxin, levodopa, trihexyphenidyl, baclofen and clonazepam are considered the treatment of choice. ${ }^{12,16}$ There are no specific studies for the treatment of movement disorders in A-T. In most cases, treatment is symptomatic and designed to reduce symptoms and improve the quality of life. Consequently, the optimal management of patients remains unclear. We observed dramatic improvement with localized botulinum toxin injections in our patient.
Clinicians should be aware of variant A-T when investigating dystonia with unknown etiology. Elevated serum alpha-fetoprotein level can be a low-cost useful screening tool. It is important to recognize variant form of $\mathrm{A}-\mathrm{T}$, as patients can avoid radiation exposures unnecessarily, as well as symptoms can improve with treatment. Therefore, A-T should be considered in a case of laryngeal dystonia, even without ataxia or telangiectasia. 


\section{REFERENCES}

1. Meijer IA, Pearson TS. The twists of pediatric dystonia: phenomenology, classification, and genetics. Semin Pediatr Neurol 2018; 25: 65-74.

2. Defazio G, Albanese A, Pellicciari R, et al. Expert recommendations for diagnosing cervical, oromandibular, and limb dystonia. Neurol Sci 2019; 40: 89-95.

3. Bertucco M, Sanger TD. Current and emerging strategies for treatment of childhood dystonia. J Hand Ther 2015; 28: 185-194.

4. Wilder-Smith E, Tan EK, Law HY, Zhao Y, Ng I, Wong MC. Spinocerebellar ataxia type 3 presenting as an L-DOPA responsive dystonia phenotype in a Chinese family. J Neurol Sci 2003; 213: 25-28.

5. Cangül H, Ozdemir O, Yakut T, et al. Pantothenate kinase-associated neurodegeneration (PKAN): molecular confirmation of a Turkish patient with a rare frameshift mutation in the coding region of the PANK2 gene. Turk J Pediatr 2009; 51: 161-165.

6. Charlesworth G, Mohire MD, Schneider SA, Stamelou M, Wood NW, Bhatia KP. Ataxia telangiectasia presenting as dopa-responsive cervical dystonia. Neurology 2013; 81: 1148-1151.

7. Scott SP, Bendix R, Chen P, Clark R, Dork T, Lavin MF. Missense mutations but not allelic variants alter the function of ATM by dominant interference in patients with breast cancer. Proc Natl Acad Sci U S A 2002; 99: 925-930.

8. Rothblum-Oviatt C, Wright J, Lefton-Greif MA, McGrath-Morrow SA, Crawford TO, Lederman HM. Ataxia telangiectasia: a review. Orphanet J Rare Dis 2016; 11: 159.
9. Ulusoy E, Edeer-Karaca N, Özen S, et al. An unusual manifestation: papillary thyroid carcinoma in a patient with ataxia-telengiectasia. Turk J Pediatr 2016; 58: 442-445.

10. Verhagen MMM, Last JI, Hogervorst FBL, et al. Presence of ATM protein and residual kinase activity correlates with the phenotype in ataxiatelangiectasia: a genotype-phenotype study. Hum Mutat 2012; 33: 561-571.

11. Teive HAG, Moro A, Moscovich M, et al. Ataxiatelangiectasia - A historical review and a proposal for a new designation: ATM syndrome. J Neurol Sci 2015; 355: 3-6.

12. Nakayama T, Sato $Y$, Uematsu M, et al. Myoclonic axial jerks for diagnosing atypical evolution of ataxia telangiectasia. Brain Dev 2015; 37: 362-365.

13. Termsarasab P, Yang AC, Frucht SJ. Myoclonus in ataxia-telangiectasia. Tremor Other Hyperkinet Mov (N Y) 2015; 5: 298.

14. Eilam R, Peter Y, Elson A, et al. Selective loss of dopaminergic nigro-striatal neurons in brains of Atm-deficient mice. Proc Natl Acad Sci U S A 1998; 95: 12653-12656.

15. Koepp M, Schelosky L, Cordes I, Cordes M, Poewe $\mathrm{W}$. Dystonia in ataxia telangiectasia: report of a case with putaminal lesions and decreased striatal [123I] iodobenzamine binding. Mov Disord 1994; 9: 455459.

16. Rebour R, Delporte L, Revol P, et al. Dopa-responsive dystonia and gait analysis: a case study of levodopa therapeutic effects. Brain Dev 2015; 37: 643-650. 